\title{
Dental Rehabilitation of a Child with Ectodermal Dysplasia: A Case Report
}

\author{
Raed R Gholman ${ }^{1}$, Wed M Kassar² ${ }^{2}$ Omar AES El Meligy ${ }^{3}$
}

\begin{abstract}
A nine-year-old girl was presented to the dental clinic at King Abdulaziz Medical City in Jeddah with carious posterior primary teeth and discolored teeth. She was referred to a pediatric geneticist to rule out any medical conditions. She was suspected to have ectodermal dysplasia (ED). DNA testing was requested and sent to Germany to confirm the clinical findings. Afterward, full-mouth rehabilitation was done including prophy and fluoride application, composite restorations, stainless steel crowns, and pulp therapy. The treatment was planned to be carried out under local anesthesia due to the cooperative behavior of the child and the parents' request. Treatment objectives were oriented to achieve esthetic and functional rehabilitation of all carious teeth, maintain occlusion, in addition to maintaining spaces for the missing permanent teeth for future replacement. The patient became caries-free. Oral hygiene was improved which resulted in gingival inflammation resolution. Spaces were maintained and the behavior of the child was improved during recall visits from positive to definitely positive. Multidisciplinary approach in the management of oral and dental manifestations associated with ED including pediatric dentists, prosthodontists, and orthodontists is of paramount importance.
\end{abstract}

Keywords: Ectodermal dysplasia, Hypodontia, Oligodontia, Stainless steel crowns.

International Journal of Clinical Pediatric Dentistry (2019): 10.5005/jp-journals-10005-1651

\section{INTRODUCTION}

Ectodermal dysplasia (ED) is a large clinically and genetically heterogeneous group of disorders characterized by developmental defects affecting tissues of ectodermal origin including teeth, hair, nails, and sweat glands. ${ }^{1}$ Ectodermal dysplasia may be inherited in any form of several genetic patterns including X-linked, autosomaldominant, and autosomal-recessive modes. ${ }^{2}$ There are two major groups of ED according to the state of sweat glands involvement: the first one is hypohidrotic or anhidrotic (Christ-SiemensTouraine syndrome), in which the sweat glands are either absent or significantly reduced in number, the second group is hidrotic (Clouston syndrome), in which sweat glands are normal. In both types, the dentition and hair are involved similarly, in contrast to hereditary patterns of nails and sweat glands involvement which are different. ${ }^{3,4}$ The most common oral finding is hypodontia of primary and permanent dentitions, so during the developmental years of these patients, they might need prosthetic treatment. ${ }^{5}$ The most frequent prosthetic treatment is the construction of complete or partial prostheses, fixed prostheses, or prostheses over implants. ${ }^{6}$

\section{Case Description}

A nine-year-old cooperative girl attended the dental clinic at King Abdulaziz Medical City (KAMC) in Jeddah with her father seeking dental treatment. Extraoral examination revealed oval-shaped face, straight profile, average mandibular plane angle, and equal facial thirds; her hair, nails, and hands appeared normal. Intraoral examination revealed the presence of carious teeth, conical-shaped lower anterior primary teeth, and oligodontia including primary and permanent teeth as follows: primary tooth \#81 and permanent teeth $\# 17,16,15,12,27,26,25,22,36,32,31,46,42$, and 41. Ectodermal dysplasia was suspected. So, to confirm diagnosis, we referred her to a pediatric geneticist to rule out any medical condition. Clinical examination that was done by the pediatric geneticist revealed no features and manifestations of ED except for oligodontia.
${ }^{1}$ Dental Department, King Abdulaziz Medical City, Jeddah, Kingdom of Saudi Arabia

${ }^{2}$ Faculty of Dentistry, Umm Al-Qura University, Makkah, Kingdom of Saudi Arabia

${ }^{3}$ Pediatric Dentistry Department, Faculty of Dentistry, King Abdulaziz University, Jeddah, Kingdom of Saudi Arabia; Pediatric Dentistry and Dental Public Health Department, Faculty of Dentistry, Alexandria University, Alexandria, Egypt

Corresponding Author: Omar AES EI Meligy, Pediatric Dentistry Department, Faculty of Dentistry, King Abdulaziz University, Jeddah, Kingdom of Saudi Arabia; Pediatric Dentistry and Dental Public Health Department, Faculty of Dentistry, Alexandria University, Alexandria, Egypt, Phone: +966 557521584, e-mail: omeligy@kau.edu.sa

How to cite this article: Gholman RR, Kassar WM, et al. Dental Rehabilitation of a Child with Ectodermal Dysplasia: A Case Report. Int J Clin Pediatr Dent 2019;12(4):362-365.

Source of support: Nil

Conflict of interest: None

To confirm the diagnosis, he requested a DNA testing, so a blood sample was sent to Centogene: The Rare Diseases Laboratory in Germany. Results revealed that the child was diagnosed with thrombospondin type laminin $G$ domain and EAR repeats (TSPEAR)-associated disorder which is a mild form of ED caused by two-frame shift mutations. Detailed intraoral clinical (Fig. 1) and radiographic examinations (Figs 2 and 3) revealed poor oral hygiene which led to multiple occlusal, proximal, and smooth surface carious lesions, in addition to generalized plaque accumulation that covered the middle thirds of the buccal and lingual surfaces which resulted in marginal gingivitis. The overjet was $4 \mathrm{~mm}$ and overbite was $3 \mathrm{~mm}$. Well-formed "U-shaped" maxillary and mandibular dental arches were evident, in addition to a class I primary canine relationship and mesial step primary molar relationship. The panoramic, bitewing, and periapical radiographic examination confirmed the oligodontia and the carious 


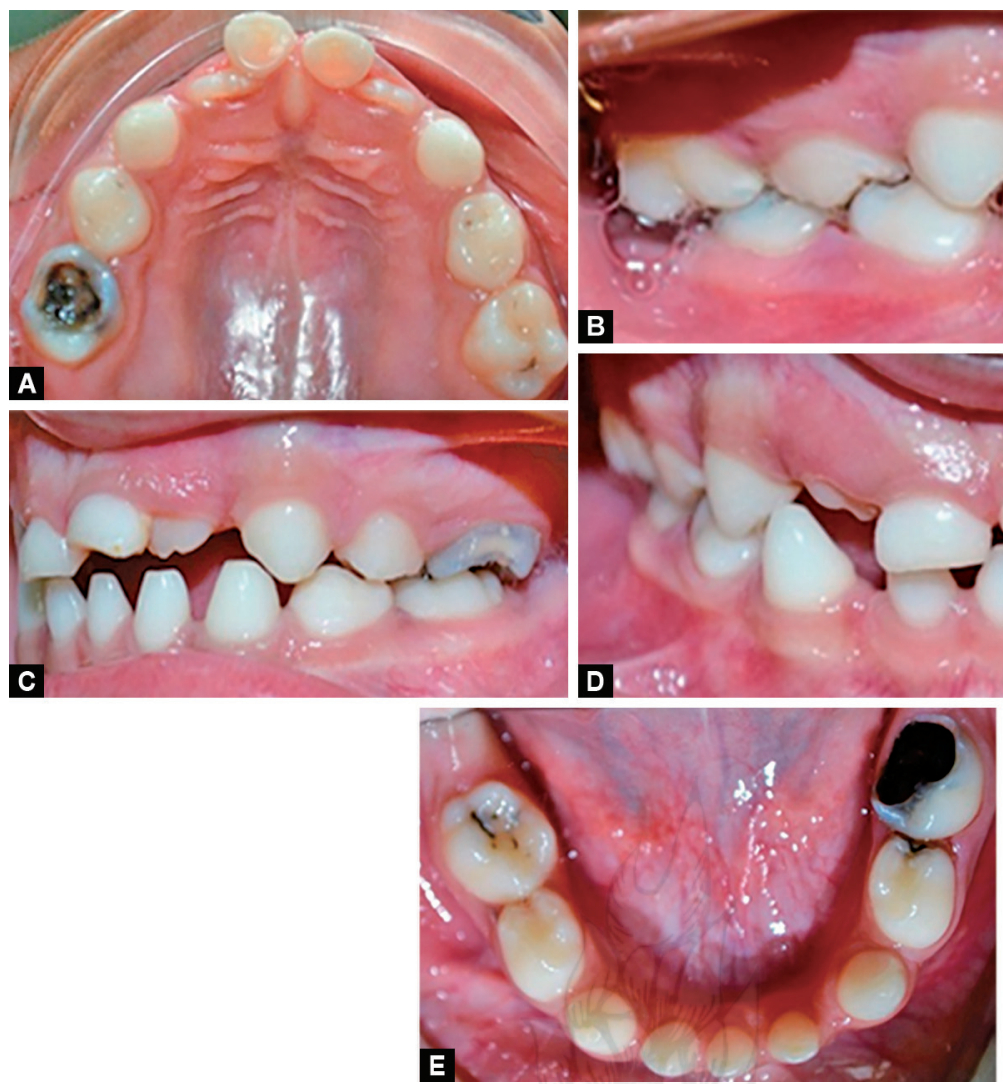

Figs $1 \mathrm{~A}$ to $\mathrm{E}$ : Intraoral preoperative photographs

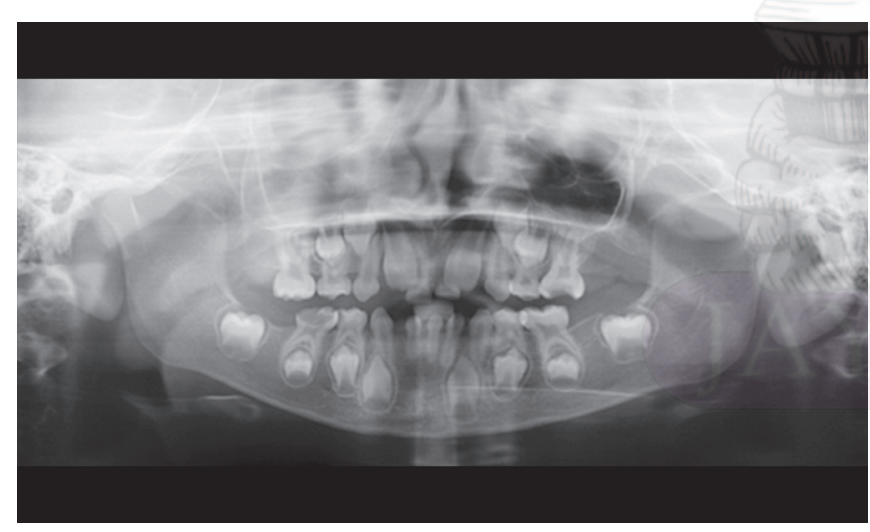

Fig. 2: Panoramic radiograph

lesion on the proximal surfaces of teeth \# $65,75,74,85$, and 84 . According to the radiographic examination, the child's dental age was delayed as compared to her chronological age which is almost nine.

\section{Discussion}

No clinical findings of ED were found except for the radiographic finding of oligodontia which is one of the features of ED. Based on the DNA testing, the child was diagnosed with ED. Caries risk was assessed regarding oral hygiene, diet, and general lifestyle. According to the caries-risk assessment (CRA), the patient was in the high risk group due to the presence of more than one interproximal carious lesion and more than three in-between meals, snacks, or sweet beverages consumption. Ectodermal dysplasia is a developmental defect which affects one or more ectodermal structures including teeth, hair, nails, and sweets glands. There are clinical and oral manifestations to ED. The clinical manifestations include loss of hair, sparse hair, thin, dry, and prone to rash skin, in addition to thick or thin, abnormally shaped or ridged nails. ${ }^{1}$ The most common oral manifestation is hypodontia, so these patients need prosthetic treatment. ${ }^{3}$ The enamel of tooth structure is derived from the ectoderm, so enamel defects might appear in patients with ED. The enamel may be softer and thinner than normal which leads to increase in cavities; also, the enamel could contain pitted surfaces. ${ }^{7}$ Saliva production may be reduced in some forms of ED leading to dry mouth which might increase the risk for tooth decay. ${ }^{7}$ The shape of the teeth may be globe-shaped and smaller than averages and often are described as being conical and tapered. In some ED, the pulp chambers are larger than normal (taurodontia). ${ }^{7}$ Delayed eruption of teeth, speech problems, and difficulty with swallowing or chewing due to missing teeth and alveolar bone deficiency may be present. ${ }^{7}$

The main goal of treatment was to restore all carious teeth in order to alleviate pain, improve esthetics, restore function, and eliminate sources of infection. The behavior management techniques that were used during the treatment were tellshow-do, distraction, positive reinforcement, and rewarding approaches. Multidisciplinary team including a pediatric dentist, prosthodontist, and orthodontist has participated in the treatment planning. The orthodontist recommended mesialization of the upper central incisors to preserve the space and bony structure. The prosthodontist recommended the construction of a removable partial denture (RPD) to replace the missing upper lateral incisors in order to hold the space for future implants. Prior to treatment, a written informed consent was obtained from the 

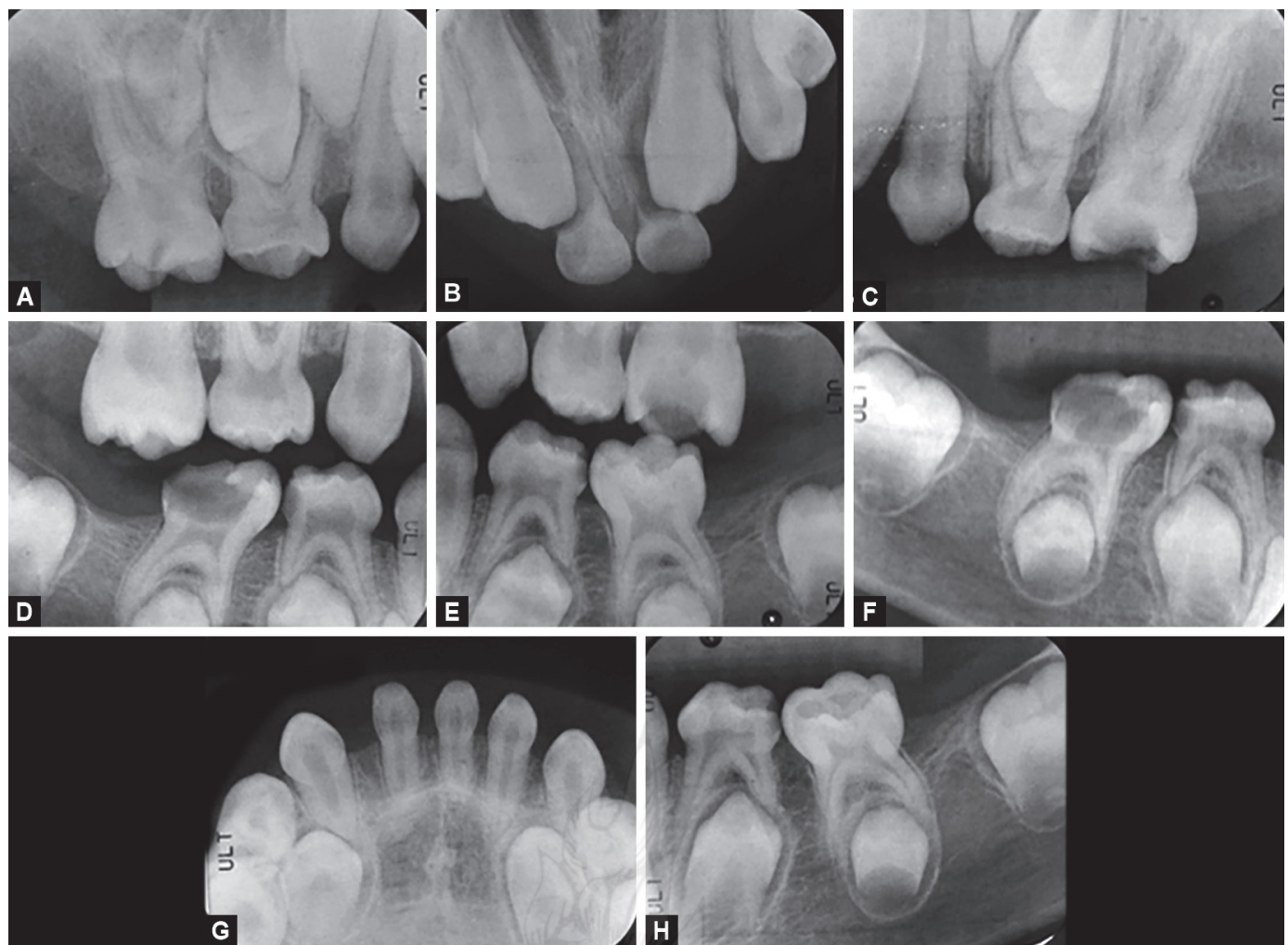

Figs $3 \mathrm{~A}$ to $\mathrm{H}$ : Full-mouth preoperative intraoral radiographs
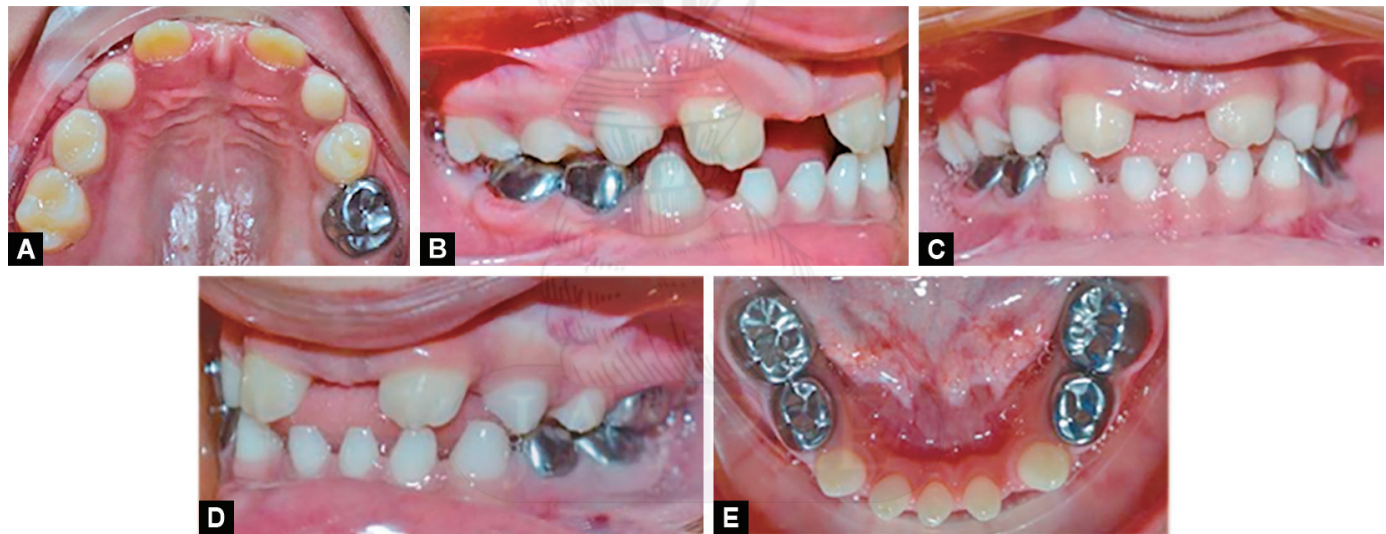

Figs $4 \mathrm{~A}$ to $\mathrm{E}$ : Three months postoperative views showing intact restorations
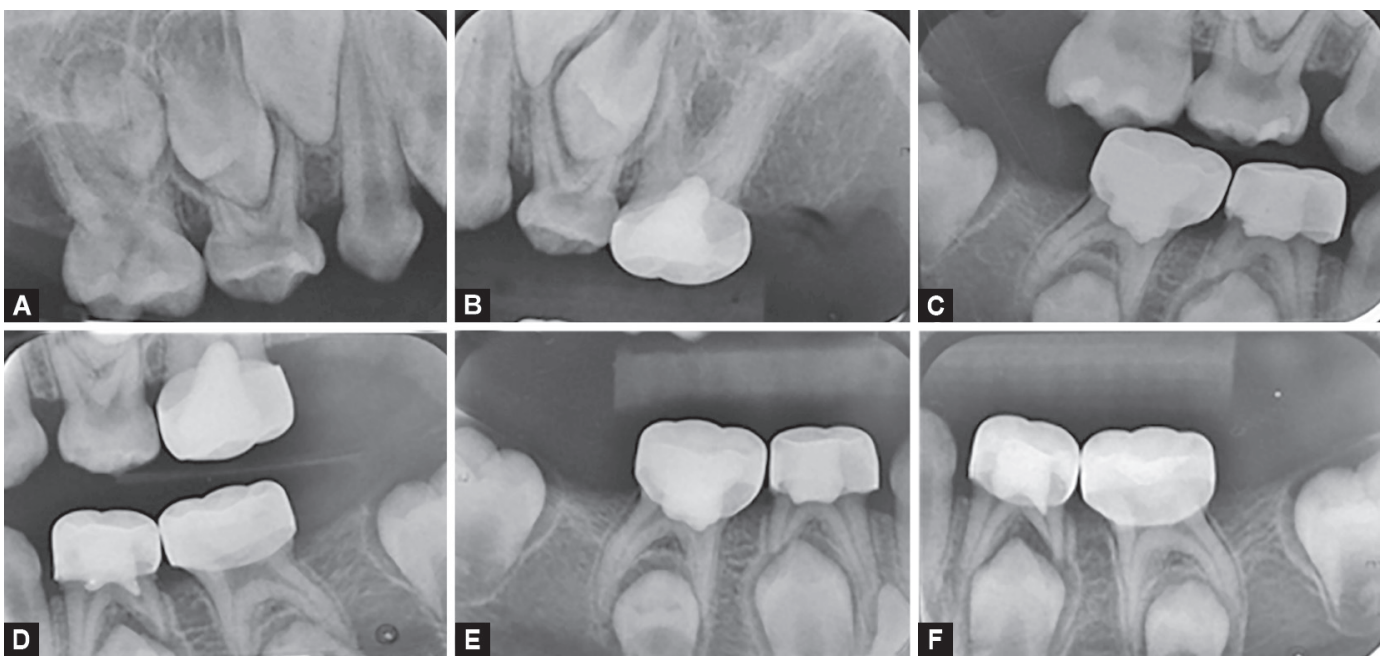

Figs $5 \mathrm{~A}$ to $\mathrm{F}$ : Postoperative intraoral radiographs 
parents. Treatment was accomplished within four visits under local anesthesia (LA).

In the 3 months recall visit, the following procedures were done: (1) the medical history and allergy status were updated; (2) the clinical examination was done with some findings such as intact restorations and fair oral hygiene with no complaint; (3) oral hygiene instructions and brushing technique were reinforced; (4) prohpylaxis and fluoride application using 5\% sodium fluoride varnish were done; (5) diet instructions were reinforced.

During the recall visit, the oral hygiene of the patient was improved and gingival inflammation was resolved. The behavior of the child has improved from positive to definitely positive and caries-free state has been achieved. Intact restorations were maintained (Fig. 4) with no complaint, in addition to insignificant radiographic findings (Fig. 5).

\section{Conclusion}

Patients diagnosed with ED require multidisciplinary treatment approach. The management of clinical manifestations presents a unique challenge for prosthodontists, orthodontists, and pediatric dentists.

\section{References}

1. Itin PH. Etiology and pathogenesis of ectodermal dysplasia. Am J Med Genet A 2014 Oct;164A(10):2472-2477. DOI: 10.1002/ajmg.a.36550.

2. Hickey AJ, Vergo Jr TJ. Prosthetic treatments for patients with ectodermal dysplasia. J Prosthet Dent 2001 Oct;86(4):364-368. DOI: 10.1067/mpr.2001.118876.

3. Tarjan I, Gabris K, et al. Early prosthetic treatment of patients with ectodermal dysplasia: a clinical report. J Prosthet Dent 2005 May;93(5):419-424. DOI: 10.1016/j.prosdent.2005.01.012.

4. Nunn JH, Carter NE, et al. The interdisciplinary management of hypodontia: background and role of paediatric dentistry. Br Dent J 2003 Mar;194(5):245-251. DOI: 10.1038/sj.bdj. 4809925.

5. Hekmatfar S, Jafari K, et al. Dental management of ectodermal dysplasia: two clinical case reports. J Dent Res Dent Clin Dent Prospects 2012 Summer;6(3):108-112. DOI: 10.5681/joddd. 2012.023.

6. Vazquez EU, Espinoza AS, et al. Prosthodontic treatment of patients afflicted with hypohidrotic ectodermal dysplasia: report of two cases. Revista Odontologica Mexicana 2017 Jan-Mar;21(1):49-53.

7. Halai T, Stevens C. Ectodermal dysplasia: a clinical overview for the dental practitioner. Dent Update 2015 Oct;42(8):779-788. DOI: 10.12968/denu.2015.42.8.779. 